\title{
Distributed Relay-Assignment Protocols for Coverage Expansion in Cooperative Wireless Networks
}

\author{
Ahmed K. Sadek, Member, IEEE, Zhu Han, Senior Member, IEEE, and K.J. Ray Liu, Fellow, IEEE
}

\begin{abstract}
One important application of cooperative communications is to extend coverage area in wireless networks without increasing infrastructure. However, a crucial challenge in implementing cooperation protocols is how to select relay-source pairs. In this paper, we address this problem based on the knowledge of the users' spatial distribution which determines the channel statistics. We consider two scenarios at the destination node, when the receiver uses MRC and when no-MRC is used. First, we characterize the optimal relay location to minimize the outage probability. Then, we propose and analyze the performance of two schemes: a distributed nearest neighbor relay assignment in which users can act as relays, and an infrastructure-based relay-assignment protocol in which fixed relay nodes are deployed in the network to help the users forward their data. The outage probabilities of these two schemes are derived. We also derive universal lower bounds on the performance of relay-assignment protocols to serve as a benchmark for our proposed protocols. Numerical results reveal significant gains when applying the proposed simple distributed algorithms over direct transmission in terms of coverage area, transmit power, and spectral efficiency. At 1 percent outage probability, more than 200 percent increase in coverage area can be achieved, $7 \mathrm{dBW}$ savings in the transmitted power, and the system can operate at $2 \mathrm{~b} / \mathrm{s} / \mathrm{Hz}$ higher spectral efficiency.
\end{abstract}

Index Terms—Relay assignment, coverage extension, cooperative diversity, wireless networks, virtual MIMO.

\section{INTRODUCTION}

T $\mathrm{T}$ is of great importance for service providers to improve 1 the coverage area in wireless networks without cost of more infrastructure and under the same quality of service requirements. This poses challenges for deployment of wireless networks because of the difficult and unpredictable nature of wireless channels. Fading, path loss, shadowing, and others are all natural impairments generally inherent in the wireless medium. Diversity techniques provide wellstudied solutions for the fading problem. Spatial diversity, especially, has gained a lot of interest after the seminal works of Foschini and Gans [1], and Telatar [2], which showed the significant performance gains that could be achieved by utilizing multiple-input-multiple-output (MIMO) systems. In wireless networks, however, installing multiple antennas at the mobile terminals might be infeasible due to cost or space limitations.

To overcome this problem, cooperative communications has been recently proposed [3], [4], [5]. The basic idea is to explore the broadcast nature of the wireless channel. In [5], Sendonaris et al. proposed a two-user-cooperation system in which every two users in the wireless network are coupled

- A.K. Sadek is with the Corporate Research and Development, Qualcomm Incorporated, 5775 Morehouse Dr., San Diego, CA 92121.

E-mail: asadek@qualcomm.com.

- Z. Han is with the Department of Electrical and Computer Engineering, University of Houston, Houston, TX 77004. E-mail: zhan2@mail.uh.edu.

- K.J. Ray Liu is with the Department of Electrical and Computer Engineering, and Institute for Systems Research, University of Maryland, College Park, MD 20742. E-mail: kjrliu@eng.umd.edu.

Manuscript received 4 May 2008; revised 26 Nov. 2008; accepted 25 June 2009; published online 29 July 2009.

For information on obtaining reprints of this article, please send e-mail to: tmc@computer.org, and reference IEEECS Log Number TMC-2008-06-0223. Digital Object Identifier no. 10.1109/TMC.2009.132.

1536-1233/10/\$26.00 (c) 2010 IEEE to help each other forming a distributed two-antenna system. Laneman et al. [3] proposed different cooperative diversity protocols for single-relay scenarios and analyzed their outage performance. Specifically, the authors in [3] proposed fixed and adaptive relaying protocols. Examples of fixed relaying are amplify-and-forward and decode-andforward relaying. Adaptive relaying protocols comprise selection relaying, in which the relay applies threshold tests on the measured channel state information to decide whether to transmit or not, and incremental relaying, in which limited feedback from the destination is employed in the form of automatic repeat request (ARQ). Distributed space-time codes for relay networks were studied in [4], [6]. In recent works [7], [8], [9], theoretical characterizations for the symbol error rate performance of a class of multinode cooperative protocols were presented. These works also provided optimal power allocation for the multinode relay problem based on an approximate expression for the symbol error rate. A practical ARQ scheme for implementing node cooperation has been recently proposed in [11] in which a stop and wait protocol is utilized, and significant gains in terms of throughput and delay have been demonstrated. Cooperative communications is a new communication paradigm that will impact the design of future wireless networks [12], [13].

In the previously cited works, the cooperating relays are assumed to exist and are already coupled with the source nodes in the network. These works also assumed a deterministic network topology, i.e., deterministic channel gain variances between different nodes in the network. If the random users' spatial distribution and the associated propagation path losses between different nodes in the 


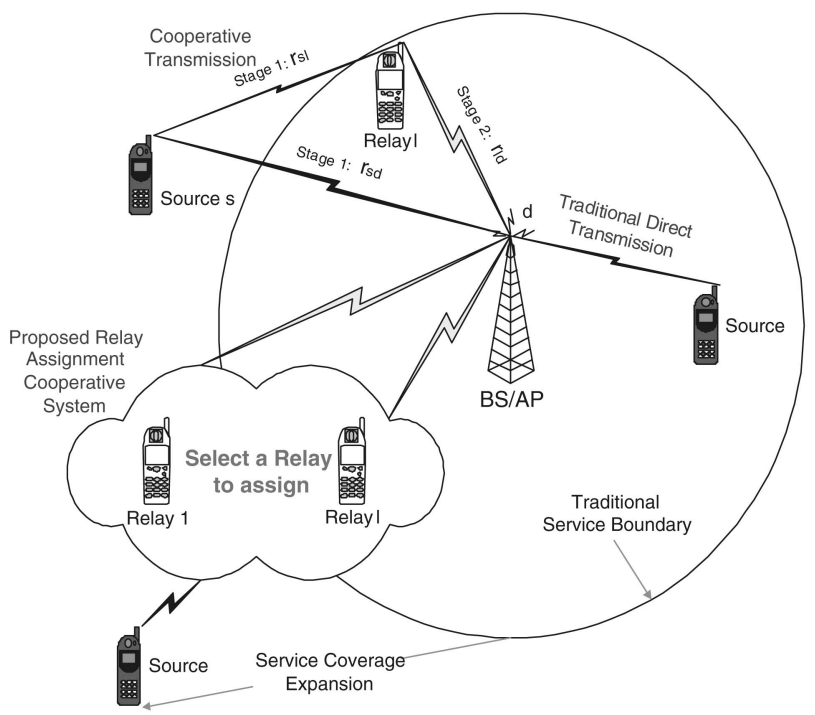

Fig. 1. Illustrating the difference between the direct and cooperative transmission schemes, and the coverage extension prospected by cooperative transmission.

network are taken into consideration, then these assumptions, in general, are no longer valid.

In this paper, we address the relay-assignment problem for implementing cooperative diversity protocols to extend coverage area in wireless networks, as depicted in Fig. 1. We study the problem under the knowledge of the users' spatial distribution which determines the channel statistics: the variance of the channel gain between any two nodes is a function of the distance between these two nodes. We consider an uplink scenario where a set of users is trying to communicate to a base station (BS) or access point (AP). A hybrid version of the incremental and selection relaying protocols proposed in [3] is developed. In this protocol, the relay only forwards the source's message if the relay decoded this message correctly and it receives a negative acknowledgement of reception from the destination. We further consider two decoding possibilities at the destination. The destination can coherently combine the signals transmitted from the source and relay in the two transmission phases, or it can simply use the received relay signal from the second transmission phase as a detection statistic. The second scenario might be more practical when it is difficult to store an analog version of the source's transmission in the first phase to combine with the relay's transmission later.

Based on the derived outage probability performance, we characterize the optimal relay location for the two receiving strategies. We then propose two practical schemes for relay assignment. The first is a simple distributed relayassignment protocol in which users can act as relays, and we refer to this protocol as the nearest-neighbor protocol. In this protocol, the helping user (relay) is chosen to be the nearest neighbor to the source. Although this choice might not be optimal in all scenarios, it is very simple to implement in a distributed manner and can achieve good performance as we will demonstrate later. The second scheme that we propose is an infrastructure-based protocol in which relay nodes are deployed in the network to help the users forward their data. Each user is assigned to its nearest relay. The role of the relays is to listen to the users' transmission, and later forward them to the destination according to the proposed relaying strategy. The relays can thus be very simple and their cost is very small compared to the cost of adding new base stations. We analyze the outage performance of the proposed protocols and further develop universal lower bounds on the performance of any relayassignment protocol to use as a benchmark for the performance of our proposed protocols.

Our numerical results show significant gains of employing the proposed protocols over direct transmission. For example, at 1 percent outage probability, more than 200 percent increase in coverage area can be achieved, $7 \mathrm{dBW}$ savings in the transmitted power, and the system can operate at $2 \mathrm{~b} / \mathrm{s} / \mathrm{Hz}$ higher spectral efficiency. The simulations also reveal the interesting result that cooperative communications can be inferior to direct transmission for large cell size, which has the same intuitive explanation as cooperation being inferior to direct transmission in high spectral efficiency regimes.

\subsection{Contributions}

The contributions of this paper can be summarized as follows:

- The outage probabilities of a hybrid version of the incremental relaying protocol and selection relaying protocol are derived.

- The optimal relay location is derived from the outage probability expression of the relaying protocol.

- A distributed relay-assignment protocol is developed in which users' terminals can act as relays. Nearest-neighbor users are selected as relays.

- Outage probability expressions for the proposed distributed relay-assignment protocol are derived assuming terminals are randomly dropped in a given area with a uniform distribution.

- An infrastructure-based relay-assignment protocol is developed. In this case, relay nodes are deployed in the network to help other user terminals communicate with the destination. The BS and deployed relays emulate a virtual distributed MIMO system with the difference that the antennas (relays) are not collocated at the BS.

- Outage probability expressions for the developed infrastructure-based relay-assignment protocol are derived.

- A universal Genie-aided lower bound is developed for the performance of a general relay-assignment protocol whether distributed or infrastructure-based. This lower bound can be used as a benchmark for the performance of any relay-assignment protocol.

- The developed relay-assignment protocols offer significant performance gains in terms of network coverage and power efficiency. This is confirmed through numerical evaluation.

\subsection{Related Work}

Related work for relay assignment assumes the availability of a list of candidate relays and develops relay-selection 
algorithms from among the list. In [10], two approaches for selecting a best relay are provided: Best-Select in the Neighbor Set and Best-Select in the Decoded Set. The BestSelect in the Neighbor Set algorithm is based on the average received SNRs, or equivalently the distance, while the latter is based on the instantaneous channel fading realization. Our proposed protocols do not assume a given candidate list to search for the best relay; instead, we assume a random node distribution across the network and take this into consideration when analyzing the performance. The performance analysis provides guidelines for the implementation of the proposed protocols.

An algorithm that selects the best relay among $M$ relays based on instantaneous channel state information and channel reciprocity has been developed in [14]. In [15], a power-aware relay-selection algorithm is described for an 802.11-based network where RTS-CTS messages are used to select the best relay based on instantaneous channel information. In [16], a subset of a group of relays is selected based on which relays decode the source's message correctly in the first phase. A second selection algorithm is also described in which the destination feedbacks a threshold to all relays, and only relays with relay-destination channel gain larger than the threshold transmit in the second phase. In [18], two relay-selection schemes are analyzed. In the first scheme, the relay is preassigned to each source prior to network deployment. In the second scheme, the source selects one relay randomly from the set of relays that decoded the source message correctly in the first phase. In all of these works, relay selection relies on instantaneous channel information knowledge at the source and the relay. Instantaneous channel information, in general, requires high overhead in terms of the signaling required for channel estimation and feedback. In our proposed protocols, relay selection only depends on channel statistics which does not vary quickly compared to channel realization.

An interesting algorithm is developed in [17] that can achieve optimal relay selection in polynomial time. This algorithm is developed for an ad hoc network with multiple source-destination pairs competing for the same pool of relay nodes. The problem is to assign relays to the sourcedestination pairs such that some cost function of the link capacities is maximized (maxmin). In our work, we are considering a different network deployment where we consider a multiple access channel with multiple sources and a single destination. One relay node can serve multiple sources in orthogonal time/frequency slots.

The rest of the paper is organized as follows: In Section 2, we present the system and channel model. In Section 3, we derive the conditional outage probability of cooperative communications and characterize the optimal relay location. The proposed relaying strategies are discussed in Section 4. Simulation results are conducted in Section 5, and finally, conclusions are drawn in Section 6.

\section{System Model}

We consider a wireless network with a circular cell of radius $\rho$. The BS/AP is located at the center of the cell, and $N$ users are uniformly distributed within the cell. The probability density function of the user's distance $r$ from the $\mathrm{BS} / \mathrm{AP}$ is thus given by

$$
q(r)=\frac{2 r}{\rho^{2}}, \quad 0 \leq r \leq \rho,
$$

and the user's angle is uniformly distributed between $[0,2 \pi)$. Two communications schemes are going to be examined in the sequel. Noncooperative transmission, or direct transmission, where users transmit their information directly to the $\mathrm{BS} / \mathrm{AP}$, and cooperative communications, where users can employ a relay to forward their data, see Fig. 1.

In the direct transmission scheme, which is employed in current wireless networks, the signal received at the destination $d$ (BS/AP) from source user $s$ can be modeled as

$$
y_{s d}=\sqrt{P K r_{s d}^{-\alpha}} h_{s d} x+n_{s d}
$$

where $P$ is the transmitted signal power, $x$ is the transmitted data with unit power, and $h_{s d}$ is the channel fading gain between the two terminals. The channel fade of any link is modeled throughout the paper as a zero-mean circularly symmetric complex Gaussian random variable with unit variance. In (2), $K$ is a constant that depends on the antennas design, $\alpha$ is the path loss exponent, and $r_{s d}$ is the distance between the two terminals. $K, \alpha$, and $P$ are assumed to be the same for all users. The term $n_{s d}$ in (2) denotes additive noise. All the noise components throughout the paper are modeled as white Gaussian noise (AWGN) with variance $N_{o}$. From (2), the received signalto-noise ratio (SNR) is

$$
\operatorname{SNR}\left(r_{s d}\right)=\frac{\left|h_{s d}\right|^{2} K r_{s d}^{-\alpha} P}{N_{o}} .
$$

In this paper, we characterize the system performance in terms of outage probability. Outage is defined as the event that the received SNR falls below a certain threshold $\gamma_{n c}$, where the subscript $n c$ denotes noncooperative transmission. The probability of outage $\mathcal{P}_{n c}$ for noncooperative transmission is defined as

$$
\mathcal{P}_{n c}=\mathcal{P}\left(\operatorname{SNR}(r) \leq \gamma_{n c}\right)
$$

The SNR threshold $\gamma_{n c}$ is determined according to the application and the transmitter/receiver structure. If the received SNR is higher than the threshold $\gamma_{n c}$, the receiver is assumed to be able to decode the received message with negligible probability of error. If an outage occurs, the packet is considered lost.

For the cooperation protocol, a hybrid version of the incremental and selection relaying proposed in [3] is employed. In this hybrid protocol, if a user's packet is lost, the BS/AP broadcasts negative acknowledgement (NACK), so that the relay assigned to this user can retransmit this packet again. This introduces spatial diversity because the source message can be transmitted via two independent channels, as depicted in Fig. 1. The relay will only transmit the packet if it is capable of capturing the packet, i.e., if the received SNR at the relay is above the threshold. In practice, this can be implemented by utilizing a cyclic redundancy check (CRC) code in the transmitted packet. The signal received from the source to the destination $d$ and the relay $l$ in the first stage can be modeled as 
$y_{s d}=\sqrt{P K r_{s d}^{-\alpha}} h_{s d} x+n_{s d}$ and $y_{s l}=\sqrt{P K r_{s l}^{-\alpha}} h_{s l} x+n_{s l}$.

If the SNR of the signal received at the destination from the source falls below the cooperation SNR threshold $\gamma_{c}$, the destination requests a second copy from the relay. Then, if the relay was able to receive the packet from the source correctly, it forwards it to the destination

$$
y_{l d}=\sqrt{P K r_{l d}^{-\alpha}} h_{l d} x+n_{l d} .
$$

The destination will then combine the two copies of the message $x$ as follows:

$$
y_{d}=a_{s d} y_{s d}+a_{l d} y_{l d},
$$

where $a_{s d}=\mathrm{I} \sqrt{P K r_{s d}^{-\alpha}} h_{s d}^{*}$ and $a_{r d}=\sqrt{P K r_{l d}^{-\alpha}} h_{l d}^{*}$. The formulation in (7) allows us to consider two scenarios at the destination: If $I=1$, then the combining at the destination is a maximal-ratio-combiner (MRC), where the destination coherently combines the signals transmitted from the source in the direct transmission phase and from the relay in the cooperation phase. On the other hand, if $\mathrm{I}=0$, then the destination only uses the relay message for decoding in the second phase if the source's message was not decoded correctly in the direct transmission phase.

\section{Relay Assignment: Protocols and Analysis}

In this section, we start with deriving the average outage for direct transmission. Then, we calculate the conditional outage probability for cooperative transmission and try to use the derived outage expressions to determine the best relay location.

\subsection{Direct Transmission}

As discussed before, the outage is defined as the event that the received SNR is lower than a predefined threshold which we denote by $\gamma_{n c}$. The outage probability for the direct transmission mode $\mathcal{P}_{O D}$ conditioned on the user's distance can be calculated as

$$
\mathcal{P}_{O D}\left(r_{s d}\right)=\mathcal{P}\left(\operatorname{SNR}\left(r_{s d}\right) \leq \gamma_{n c}\right)=1-\exp \left(-\frac{N_{o} \gamma_{n c} r_{s d}^{\alpha}}{K P}\right),
$$

where the above follows because $\left|h_{s d}\right|^{2}$, the magnitude square of the channel fade, has an exponential distribution with unit mean. At high SNR, (8) can be approximated as $\frac{N_{o} \gamma_{n c} r_{s d}^{\alpha}}{K P}$.

To find the average outage probability over the cell, we need to average over the user distribution in (1). The average outage probability is thus given by

$$
\begin{aligned}
\mathcal{P}_{O D} & =\int_{0}^{\rho} \mathcal{P}_{O D}\left(r_{s d}\right) q\left(r_{s d}\right) d r_{s d} \\
& =\int_{0}^{\rho} \frac{2 r_{s d}}{\rho^{2}}\left(1-\exp \left(-\frac{N_{o} \gamma_{n c} r_{s d}^{\alpha}}{K P}\right)\right) d r_{s d} \\
& =1-\frac{2}{\alpha \rho^{2}}\left(\frac{K P}{N_{o} \gamma_{n c}}\right)^{\frac{2}{\alpha}} \Gamma\left(\frac{2}{\alpha}, \frac{N_{o} \gamma_{n c} \rho^{\alpha}}{K P}\right) \simeq \frac{2 \gamma_{n c} \rho^{\alpha} N_{o}}{K P(\alpha+2)}
\end{aligned}
$$

where $\Gamma(\cdot, \cdot)$ is the incomplete Gamma function which is defined as [23]

$$
\Gamma(a, x)=\int_{0}^{x} \exp ^{-t} t^{a-1} d t
$$

\subsection{Cooperative Transmission: Conditional Outage Probability}

Consider a source-destination pair that is $r_{s d}$ units distance apart. Let us compute the conditional outage probability for given locations of the user and the helping relay. Using (5), the SNRs received at the BS/AP $d$ and the relay $l$ from the source $s$ are given by

$\operatorname{SNR}\left(r_{s d}\right)=\frac{\left|h_{s d}\right|^{2} K r_{s d}^{-\alpha} P}{N_{o}}, \quad$ and $\quad \operatorname{SNR}\left(r_{s l}\right)=\frac{\left|h_{s l}\right|^{2} K r_{s l}^{-\alpha} P}{N_{o}}$

While from (7), the SNR of the combined signal received at the $\mathrm{BS} / \mathrm{AP}$ is given by

$$
\mathrm{SNR}_{d}=\mathrm{I} \frac{\left|h_{s d}\right|^{2} K r_{s d}^{-\alpha} P}{N_{o}}+\frac{\left|h_{l d}\right|^{2} K r_{l d}^{-\alpha} P}{N_{o}} .
$$

The terms $\left|h_{s d}\right|^{2},\left|h_{s l}\right|^{2}$, and $\left|h_{l d}\right|^{2}$ are mutually independent exponential random variables with unit mean. The outage probability of the cooperative transmission $P_{O C}$ conditioned on the fixed topology of the user $s$ and the relay $l$ can be calculated as follows: Using the law of total probability, we have

$$
\mathcal{P}_{O C}=\operatorname{Pr}\left(\text { Outage } \mid \mathrm{SNR}_{s d} \leq \gamma_{c}\right) \operatorname{Pr}\left(\mathrm{SNR}_{s d} \leq \gamma_{c}\right),
$$

where the probability of outage is zero if $\mathrm{SNR}_{s d}>\gamma_{c}$. The outage probability conditioned on the event that the sourcedestination link is in outage is given by

$$
\begin{aligned}
\operatorname{Pr}\left(\text { Outage } \mid \mathrm{SNR}_{s d} \leq \gamma_{c}\right)= & \operatorname{Pr}\left(\mathrm{SNR}_{s l} \leq \gamma_{c}\right)+\operatorname{Pr}\left(\mathrm{SNR}_{s l}\right. \\
& \left.>\gamma_{c}\right) \operatorname{Pr}\left(\mathrm{SNR}_{d} \leq \gamma_{c} \mid \mathrm{SNR}_{s d} \leq \gamma_{c}\right),
\end{aligned}
$$

where the addition of the above probabilities is because they are disjoint events, and the multiplication is because the source-relay link is assumed to fade independently from the other links. The conditioning was removed for the same reason.

For the case where MRC is allowed at the destination, then the conditional outage probability at the destination is given by

$$
\operatorname{Pr}\left(\mathrm{SNR}_{d} \leq \gamma_{c} \mid \mathrm{SNR}_{s d} \leq \gamma_{c}\right)=\frac{\operatorname{Pr}\left(\mathrm{SNR}_{d} \leq \gamma_{c}\right)}{\operatorname{Pr}\left(\mathrm{SNR}_{s d} \leq \gamma_{c}\right)}
$$

Using (15) and (14) in (13), the conditional outage probability for cooperative communications with MRC can be calculated as

$$
\begin{aligned}
\mathcal{P}_{O C}\left(r_{s d}, r_{s l}, r_{l d}\right)= & \left(1-f\left(\gamma_{c}, r_{s d}\right)\right)\left(1-f\left(\gamma_{c}, r_{s l}\right)\right) \\
& +f\left(\gamma_{c}, r_{s l}\right)\left[1-\frac{r_{s d}^{-\alpha}}{r_{s d}^{-\alpha}-r_{l d}^{-\alpha}} f\left(\gamma_{c}, r_{s d}\right)\right. \\
& \left.-\frac{r_{l d}^{-\alpha}}{r_{l d}^{-\alpha}-r_{s d}^{-\alpha}} f\left(\gamma_{c}, r_{l d}\right)\right]
\end{aligned}
$$

where, $f(x, y)=\exp \left(-\frac{N_{o} x y^{\alpha}}{K P}\right)$. The above expression can be simplified as follows: 


$$
\begin{gathered}
\mathcal{P}_{O C}\left(r_{s d}, r_{s l}, r_{l d}\right)=\left[1-f\left(\gamma_{c}, r_{s d}\right)\right]-\frac{r_{l d}^{-\alpha}}{r_{l d}^{-\alpha}-r_{s d}^{-\alpha}} f\left(\gamma_{c}, r_{s l}\right) \\
{\left[f\left(\gamma_{c}, r_{l d}\right)-f\left(\gamma_{c}, r_{s d}\right)\right] .}
\end{gathered}
$$

For the $\mathrm{I}=1$ case, or when MRC is used at the destination, then using the approximation $\exp (-x) \simeq 1-x+\frac{x^{2}}{2}$ for small $x$, the above outage expression can be approximated at high SNR to

$$
\begin{aligned}
\mathcal{P}_{O C}\left(r_{s d}, r_{s l}, r_{l d}\right) \simeq & \frac{N_{o}}{K P} r_{s d}^{\alpha}-\frac{N_{o}^{2}}{2 K^{2} P^{2}} r_{s d}^{2 \alpha}-\frac{r_{s d}^{\alpha}}{r_{s d}^{\alpha}-r_{l d}^{\alpha}} \\
& \times\left[\frac{N_{o}}{K P}\left(r_{s d}^{\alpha}-r_{l d}^{\alpha}\right)+\frac{N_{o}^{2}}{2 K^{2} P^{2}}\right. \\
& \left.\left(\left(r_{l d}^{\alpha}-r_{s d}^{\alpha}\right)\left(2 r_{s l}^{\alpha}+r_{l d}^{\alpha}+r_{s d}^{\alpha}\right)\right)\right] .
\end{aligned}
$$

Simplifying the above expression, we get

$$
\mathcal{P}_{O C}\left(r_{s d}, r_{s l}, r_{l d}\right) \simeq \frac{N_{o}^{2}}{2 K^{2} P^{2}} r_{s d}^{2 \alpha}\left[2 \frac{r_{s l}^{\alpha}}{r_{s d}^{\alpha}}+\frac{r_{l d}^{\alpha}}{r_{s d}^{\alpha}}\right] .
$$

For the $\mathrm{I}=0$ case, or when no-MRC is used at the destination, then the conditional outage expression in (15) simplifies to

$$
\operatorname{Pr}\left(\mathrm{SNR}_{d} \leq \gamma_{c} \mid \mathrm{SNR}_{s d} \leq \gamma_{c}\right)=\operatorname{Pr}\left(\mathrm{SNR}_{l d} \leq \gamma_{c}\right) .
$$

This is because the SNR received at the destination in this case is just due to the signal received from the relaydestination path. The conditional outage expression in this case can be shown to be given by

$\mathcal{P}_{O C}\left(r_{s d}, r_{s l}, r_{l d}\right)=\left(1-f\left(\gamma_{c}, r_{s d}\right)\right)\left[1-f\left(\gamma_{c}, r_{l d}\right) f\left(\gamma_{c}, r_{s l}\right)\right]$.

\subsection{Optimal Relay Position}

To find the optimal relay position, we need to find the pair $\left(r_{s l}, r_{l d}\right)$ that minimizes the conditional outage probability expression in (17). First, we consider the $\mathrm{I}=1$ scenario, where MRC is utilized at the receiver.

\subsubsection{MRC Case}

Examining the conditional outage expression in (17), it is clear that for any value of $r_{l d}$, the optimal value for $r_{s l}$ that minimizes the outage expression is the minimum value for $r_{s l}$. Since, for any value of $r_{l d}$ the minimum $r_{s l}$ lies on the straight line connecting the source and destination, we get the first intuitive result that the optimal relay position is on this straight line.

Now, we prove that the optimal relay position is close to the source. Normalizing with respect to $r_{s d}$ by substituting $x=\frac{r_{l d}}{r_{s d}}$ in (19) and $1-x=\frac{r_{s l}}{r_{s d}}$, we have

$$
\mathcal{P}_{O C}(x)=\frac{N_{o}^{2}}{2 K^{2} P^{2}} r_{s d}^{2 \alpha}\left[2(1-x)^{\alpha}+x^{\alpha}\right] .
$$

Taking the derivative with respect to $x$, we get

$$
\frac{\partial \mathcal{P}_{O C}(x)}{\partial x}=\frac{N_{o}^{2}}{2 K^{2} P^{2}} r_{s d}^{2 \alpha}\left[-2 \alpha(1-x)^{\alpha-1}+\alpha x^{\alpha-1}\right] .
$$

Equating the above derivative to zero, we get the unique solution

$$
x^{*}=\frac{1}{1+\left(\frac{1}{2}\right)^{\frac{1}{\alpha-1}}} .
$$

Checking for the second order conditions, we get that $\mathcal{P}_{O C}^{\prime \prime}(x) \geq 0$, which shows that the problem is convex, and $x^{*}$ specified in (24) is indeed the optimal relay position.

Note from the optimal relay position in (24) that for propagation path loss $\alpha \geq 2$, we have that $x^{*}>0.5$, which means that the optimal relay position is closer to the source node.

\subsubsection{No-MRC Case}

Next, we determine the optimal relay location for the $\mathrm{I}=0$ case. From the conditional outage expression in (21), and similar to the MRC case, the optimal relay position is on the line joining the source and destination.

We can substitute for $r_{l d}$ by $r_{s d}-r_{s l}$. The optimal relay position can be found via solving the following optimization problem:

$$
r_{s l}^{*}=\arg \min _{r_{s l}} \mathcal{P}_{O C}\left(r_{s d}, r_{s l}\right), \quad \text { subject to } \quad 0 \leq r_{s l} \leq r_{s d} .
$$

Since the minimization of the expression in (21) with respect to $r_{s l}$ is equivalent to minimizing the exponent in the second bracket, solving the optimization problem in (25) is equivalent to solving

$$
r_{s l}^{*}=\arg \min _{r_{s l}} r_{s l}^{\eta}+\left(r_{s d}-r_{s l}\right)^{\eta}, \quad \text { subject to } \quad 0 \leq r_{s l} \leq r_{s d} .
$$

The above optimization problem can be simply analytically solved, and the optimal relay position can be shown to be equal to $r_{s l}^{*}=\frac{r_{s d}}{2}$ for $\eta>1$. Therefore, the optimal relay position is exactly in the middle between the source and destination when no-MRC is used at the destination.

\section{Relay-Assignment Algorithms}

In this section, we propose two distributed relay-assignment algorithms. The first is a user-user cooperation protocol in which the nearest neighbor is assigned as a relay. The second considers the scenario where fixed relays are deployed in the network to help the users.

\subsection{Nearest-Neighbor Protocol}

In this section, we propose the Nearest-Neighbor protocol for relay assignment that is both distributed and simple to implement. In this protocol, the relay assigned to help is the nearest neighbor to the source. The source sends a "HelpRequest" message to his neighbors. Nodes that are willing to help respond with a "Ready-to-Help" message. The source then selects the node whose signal was received with the shortest arrival time and assigns this node as its relay. Since the proposed scheme is a distributed relay-assignment scheme, one node can act as a relay node for more than one source, and since we assume an orthogonal multiple access scheme, as TDMA or FDMA, there is no interference between different source transmissions. In a mobile environment, relay-assignment updates can be controlled by the frequency of transmitting the "HelpRequest" message. This is a parameter that can be set by the designer depending on the deployment scenario.

The outage probability expression, which we refer to as $\mathcal{P}_{O N N}$, for given source-relay-destination locations, is still 


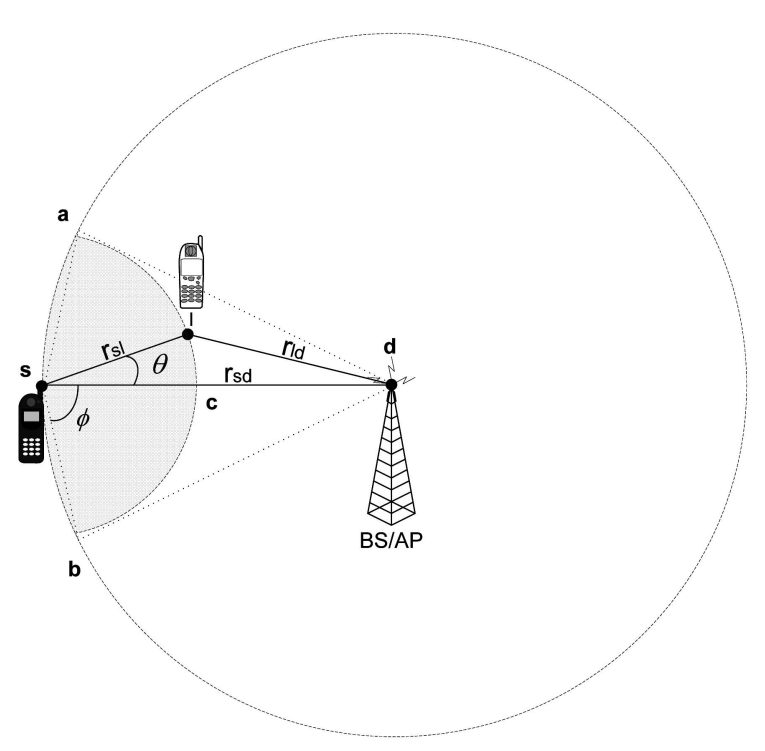

Fig. 2. Illustrating cooperation under nearest-neighbor protocol: The nearest neighbor is at a distance $r_{s l}$ from the source. Therefore, the shaded area should be empty from any users.

given by (17) for the MRC case and (21) for the no-MRC case. To find the total probability, we need to average over all possible locations of the user and the relay. The user's location distribution with respect to the BS/AP is still given as in the direct transmission case (1). The relay's location distribution, however, is not uniform. In the sequel, we calculate the probability density function of the relay's location. According to our protocol, the relay is chosen to be the nearest neighbor to the user. The probability that the nearest neighbor is at distance $r_{s l}$ from the source is equivalent to calculating the probability that the shaded area in Fig. 2 is empty.

Denote this area, which is the intersection of the two circles with centers $s$ and $d$, by $A\left(r_{s d}, r_{s l}\right)$. For $0<r_{s l} \leq$ $\rho-r_{s d}$, the area of intersection is a circle with radius $r_{s l}$ and center $s$. The probability density function of $r_{s l}, p_{r_{s l}}(x)$, can be calculated as

$$
\begin{aligned}
p_{r_{s l}}(x) & =\frac{\partial}{\partial x}\left(1-\mathcal{P}\left(r_{s l}>x\right)\right) \\
& =\frac{\partial}{\partial x}\left(1-\left(1-\frac{x^{2}}{\rho^{2}}\right)^{N-1}\right) \\
& =\frac{2(N-1) x}{\rho^{2}}\left(1-\frac{x^{2}}{\rho^{2}}\right)^{N-2}, \quad 0<r_{s l} \leq \rho-r_{s d}
\end{aligned}
$$

For $\rho-r_{s d}<r_{s l} \leq \rho+r_{s d}$, the intersection between the two circles can be divided into three areas: 1) the area of the sector $a c b$ in circle $s, 2$ ) area of the triangle $a s b$, and 3) area enclosed by the chord $a b$ in circle $d$. Hence, the intersection area, denoted by $A\left(r_{s d}, r_{s l}\right)$, can be written as

$$
A\left(r_{s d}, r_{s l}\right)=r_{s l}^{2} \theta+\frac{1}{2} r_{s l}^{2} \sin (2 \theta)+\left(\rho^{2} \phi-\frac{1}{2} \rho^{2} \sin (2 \phi)\right),
$$

where, $\theta=\cos ^{-1}\left(\frac{\rho^{2}-r_{s l}^{2}-r_{s d}^{2}}{2 r_{s l} r_{s d}}\right)$, and $\phi=\cos ^{-1}\left(\frac{r_{s l}^{2}-\rho^{2}-r_{s d}^{2}}{2 \rho r_{s d}}\right)$. The probability density function for $r_{s l}$ for this range is given by

$$
\begin{aligned}
p_{r_{s l}}(x)= & \frac{\partial}{\partial x}\left(1-\left(1-\frac{A\left(r_{s d}, r_{s l}\right)}{\pi \rho^{2}}\right)^{N-1}\right), \\
& \rho-r_{s d}<r_{s l} \leq \rho+r_{s d} .
\end{aligned}
$$

This completely defines the probability density function for the nearest neighbor and the average can be found numerically as the integrations are complex to compute analytically. Next, we derive an approximate expression for the outage probability under the following two assumptions: Since the relay is chosen to be the nearest neighbor to the source, the SNR received at the relay from the source is rarely below the threshold $\gamma_{c}$; hence, we assume that the event of the relay being in outage is negligible. The second assumption is that the nearest neighbor always lies on the intersection of the two circles, as points $a$ or $b$ in Fig. 2. This second assumption is a worst case scenario, because a relay at distance $r_{s l}$ from the source can be anywhere on the arc $\widehat{a c b}$, and a worst case scenario is to be at points $a$ or $b$. However, this simplifies the outage calculations since the conditional outage probability (17) becomes only a function of the source distance $r_{s l}$ as follows:

$$
\mathcal{P}_{O N N}\left(r_{s d}\right) \simeq 1-f\left(\gamma_{c}, r_{s d}\right)-\frac{N_{o} \gamma_{c}}{K P} r_{s d}^{\gamma} f\left(\gamma_{c}, r_{s d}\right) .
$$

Averaging (30) over the user distribution (1) and using the definition of the incomplete Gamma function in (10), we get

$$
\begin{aligned}
\mathcal{P}_{O N N} \simeq & 1-\frac{2}{\alpha \rho^{2}}\left(\frac{K P}{N_{o} \gamma_{c}}\right)^{\frac{2}{\alpha}} \Gamma\left(\frac{2}{\alpha}, \frac{N_{o} \gamma_{c} \rho^{\alpha}}{K P}\right) \\
& -\frac{2}{\alpha \rho^{2}}\left(\frac{K P}{N_{o} \gamma_{c}}\right)^{\frac{2}{\alpha}} \Gamma\left(\frac{2}{\alpha}+1, \frac{N_{o} \gamma_{c} \rho^{\alpha}}{K P}\right) .
\end{aligned}
$$

Using the same approximation as above, the conditional outage probability for the no-MRC case is given by

$$
\mathcal{P}_{\text {ONN }}\left(r_{s d}\right)=\left(1-f\left(\gamma_{c}, r_{s d}\right)\right)^{2} .
$$

Averaging the above expression over $r_{s d}$, we have

$$
\begin{aligned}
\mathcal{P}_{O N N} \simeq & 1-\frac{4}{\alpha \rho^{2}}\left(\frac{K P}{N_{o} \gamma_{c}}\right)^{\frac{2}{\alpha}} \Gamma\left(\frac{2}{\alpha}, \frac{N_{o} \gamma_{c} \rho^{\alpha}}{K P}\right) \\
& +\frac{2}{\alpha \rho^{2}}\left(\frac{K P}{2 N_{o} \gamma_{c}}\right)^{\frac{2}{\alpha}} \Gamma\left(\frac{2}{\alpha}, \frac{2 N_{o} \gamma_{c} \rho^{\alpha}}{K P}\right) .
\end{aligned}
$$

\subsection{Infrastructure-Based Relay Assignment}

The proposed nearest-neighbor protocol, although simple to implement, requires that users are willing to cooperate. In wireless networks in which users belong to different authorities, users might act selfishly to maximize their own gains. For such scenarios, protocols for enforcing cooperation, or to introduce incentives for the users to cooperate, need to be implemented. In this section, we propose another solution to this problem. We propose deploying dummy nodes in the network that act as relays and do not have their own data to transmit. Each user will be associated with one relay to help in forwarding the dropped packets. The user can select the closest relay, which can be implemented using the exchange of "Hello" messages and selecting the signal with shortest arrival time, for example.

Continuing with our circular model for the cell, with uniform users' distribution, the relays are deployed 


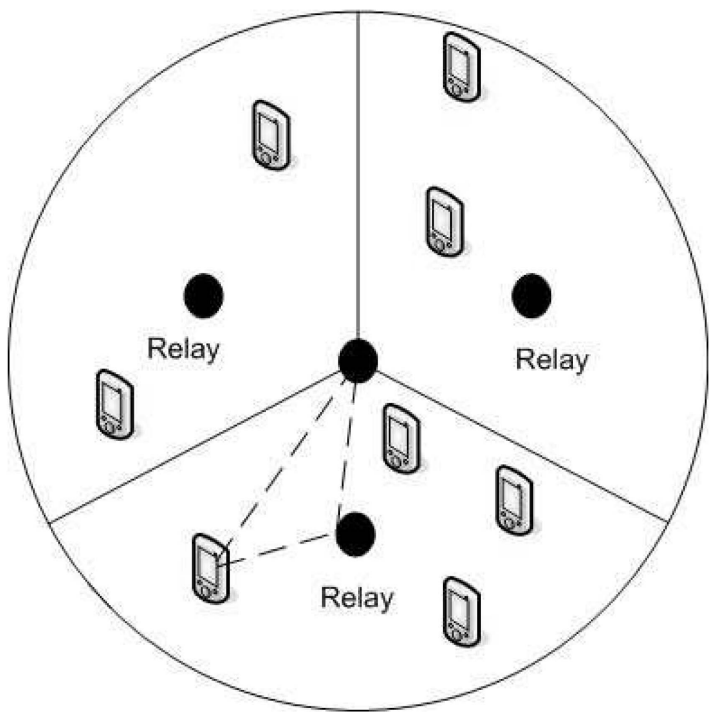

Fig. 3. Illustrating cooperation under the infrastructure-based protocol.

uniformly by dividing the cell into a finite number $m$ of equal sectors, equal to the number of fixed relays to be deployed. Fig. 3 depicts a network example for $m=3$. The relays are deployed at a distance $r_{l d}$ from the destination. This distance should be designed to minimize the average outage probability as follows:

$$
r_{l d}^{*}=\arg \min \mathcal{P}_{O C}\left(r_{l d}\right), \quad \text { s.t. } 0<r_{l d}<\rho,
$$

where the average outage probability $\mathcal{P}_{O C}\left(r_{l d}\right)$ is defined as

$$
\mathcal{P}_{O C}=\int_{0}^{\rho} \frac{2 l_{s d}}{\rho^{2}} \int_{-\frac{\pi}{m}}^{\frac{\pi}{m}} \mathcal{P}_{O C}\left(r_{s d}, r_{s l}(\theta), r_{l d}\right) \frac{m}{2 \pi} d \theta d l_{s d},
$$

where $\mathcal{P}_{O C}\left(l_{s d}, l_{s r}, l_{r d}\right)$ is defined in (17), and the distance from the source to the fixed relay is given by

$$
r_{s l}(\theta)=\sqrt{r_{s d}^{2}+r_{l d}^{2}-2 r_{s d} r_{l d} \cos (\theta)}
$$

where $\theta$ is uniformly distributed between $\left[-\frac{\pi}{m}, \frac{\pi}{m}\right]$. Solving the above optimization problem is very difficult, hence, we are going to consider the following heuristic. We are going to deploy the relay at the position that minimizes the mean square distance between the users in the sector and the relay. Without loss of generality, assuming the line dividing the sector to be the $x$-axis, the mean square distance between a user at distance $r$ and angle $\theta$ from the center of the cell and the relay is given by the following function:

$$
C\left(r_{l d}\right)=\mathrm{E}\left(\left\|r e^{j \theta}-r_{l d}\right\|^{2}\right)
$$

where $j=\sqrt{-1}$ and $\mathrm{E}$ denotes the joint statistical expectation over the random variables $r$ and $\theta$. Solving for the optimal $r_{l d}$ that minimizes $C\left(r_{l d}\right)$

$$
r_{l d}^{*}=\arg \min \mathrm{E}\left(\left\|r e^{j \theta}-r_{l d}\right\|^{2}\right),
$$

we get

$$
r_{l d}^{*}=\frac{2 m}{3 \pi} \sin \left(\frac{\pi}{m}\right) \rho
$$

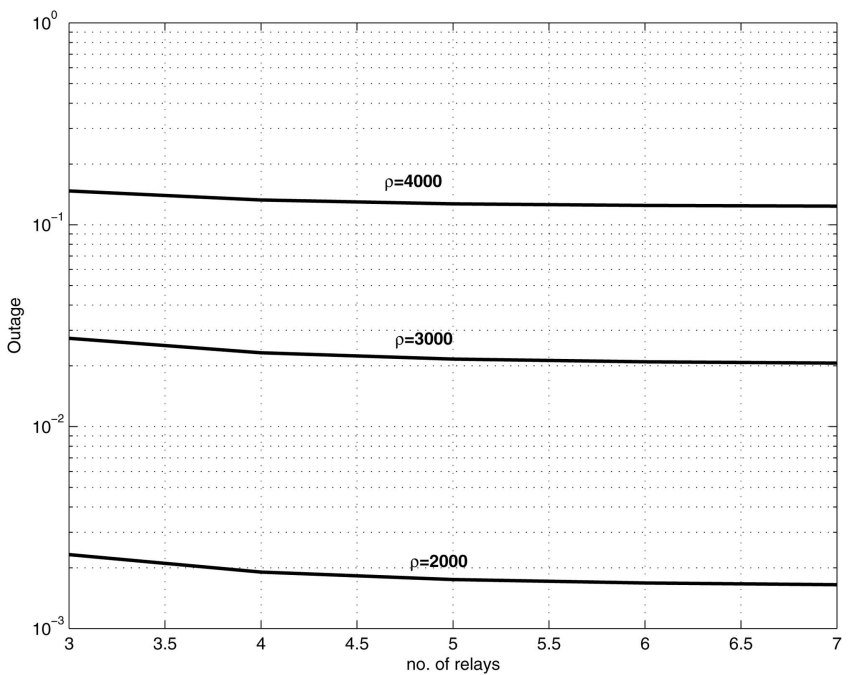

Fig. 4. Average outage probability versus the number of relays in fixed relaying.

where $\rho$ is the cell radius. Thus, in our proposed protocol, the relays are going to be deployed at a distance $r_{l d}^{*}$ from the center of the cell.

Fig. 4 depicts the average outage probability versus the number of relays deployed in the network for different cell sizes. The numerical results are for the following parameters: $K=1, \alpha=3, P=0.05, R=1$, and $N_{o}=10^{-12}$. We can see from the results that the performance saturates at approximately $m=6$ relays.

\subsection{Universal Performance Lower Bounds}

For both the MRC and no-MRC cases, we determined the optimal relay location. For the MRC case, the optimal relay position is toward the source and can be determined according to (24). For the no-MRC case, we showed that the optimal relay position is in the midpoint between the source and the destination. We will drive a lower bound on the outage probability for any relay-assignment protocol based on a Genie-aided approach. This bound serves as a benchmark for the performance of the Nearest-Neighbor protocol, and the fixed-relaying scheme proposed in the paper. The Genie-Aided protocol works as follows: For any source node in the network, a Genie is going to put a relay at the optimal position on the line joining this source node and the destination (BS/AP).

Next, we analyze the average outage performance of the Genie-Aided protocol. For the MRC case, substituting the optimal relay position in (24) in the conditional outage expression in (17) we get

$$
\begin{gathered}
\mathcal{P}_{O C}\left(r_{s d}\right)=1-f\left(\gamma_{c}, r_{s d}\right)-\frac{1}{1-x^{*}} f\left(\gamma_{c},\left(1-x^{*}\right) r_{s d}\right) \\
\left(f\left(\gamma_{c}, x^{*} r_{s d}\right)-f\left(\gamma_{c}, r_{s d}\right)\right) .
\end{gathered}
$$

Averaging the above expression over the user distribution, the average outage probability for the Genie-aided lower bound for the MRC case $I=1$ is given by 


$$
\begin{aligned}
\mathcal{P}_{O G, 1}= & -\frac{2}{\alpha \rho^{2}}\left(\frac{K P}{N_{o} \gamma_{c}}\right)^{\frac{2}{\alpha}} \Gamma\left(\frac{2}{\alpha}, \frac{N_{o} \gamma_{c} \rho^{\alpha}}{K P}\right) \\
- & \frac{1}{1-\left(x^{*}\right)^{\alpha}} \frac{2}{\alpha \rho^{2}}\left(\frac{K P}{N_{o} \gamma_{c}\left(\left(1-x^{*}\right)^{\alpha}+\left(x^{*}\right)^{\alpha}\right)}\right)^{\frac{2}{\alpha}} \\
& \Gamma\left(\frac{2}{\alpha}, \frac{\left(\left(1-x^{*}\right)^{\alpha}+\left(x^{*}\right)^{\alpha}\right) N_{o} \gamma_{c} \rho^{\alpha}}{K P}\right) \\
+ & \frac{1}{1-\left(x^{*}\right)^{\alpha} \frac{2}{\alpha \rho^{2}}\left(\frac{K P}{N_{o} \gamma_{c}\left(\left(1-x^{*}\right)^{\alpha}+1\right)}\right)^{\frac{2}{\alpha}}} \\
& \Gamma\left(\frac{2}{\alpha}, \frac{\left(\left(1-x^{*}\right)^{\alpha}+1\right) N_{o} \gamma_{c} \rho^{\alpha}}{K P}\right) .
\end{aligned}
$$

We will denote the average probability of outage for the no-MRC case by $\mathcal{P}_{O G, 2}$. Substituting the optimal relay position $r_{s l}^{*}$ in the conditional outage expression (17), we get

$$
\begin{aligned}
\mathcal{P}_{O G}\left(r_{s d}\right)= & \left(1-\exp \left(-\frac{\left.N_{o} \gamma_{c} r_{s d}^{\alpha}\right)}{K P}\right)\right) \\
& \left(1-\exp \left(-\frac{2 N_{o} \gamma_{c}\left(\frac{r_{s d}}{2}\right)^{\alpha}}{K P}\right)\right) .
\end{aligned}
$$

Averaging the above expression over all possible users' locations

$$
\begin{aligned}
\mathcal{P}_{O G, 2}= & +\frac{2}{\alpha \rho^{2}}\left(\frac{k P}{N_{o} \gamma_{c}\left(1+2^{1-\alpha}\right)}\right)^{\frac{2}{\alpha}} \Gamma\left(\frac{2}{\alpha}, \frac{N_{o} \gamma_{c}\left(1+2^{1-\alpha}\right) \rho^{\alpha}}{k P}\right) \\
& -\frac{2}{\alpha \rho^{2}}\left(\frac{k P}{N_{o} \gamma_{c}}\right)^{\frac{2}{\alpha}} \Gamma\left(\frac{2}{\alpha}, \frac{N_{o} \gamma_{c} \rho^{\alpha}}{k P}\right) \\
& -\frac{2}{\alpha \rho^{2}}\left(\frac{k P}{N_{o} \gamma_{c} 2^{1-\alpha}}\right)^{\frac{2}{\alpha}} \Gamma\left(\frac{2}{\alpha}, \frac{N_{o} \gamma_{c} 2^{1-\alpha} \rho^{\alpha}}{k P}\right) .
\end{aligned}
$$

\section{Numerical Results}

In all of our numerical results, we compare the outage performance of three different transmission schemes: direct transmission, the nearest-neighbor protocol, and the infrastructure-based relaying protocol. The channel fading between any two nodes (either a user and the BS/AP or two users) is modeled as a random Rayleigh fading channel with unit variance.

For fair comparison between the proposed cooperative schemes and the direct transmission scheme, the spectral efficiency is kept fixed in both cases and this is done as follows: Since a packet is either transmitted once or twice in the cooperative protocol, the average rate in the cooperative case can be calculated as

$$
\mathrm{E}\left(R_{c}\right)=R_{c} \mathcal{P}_{O D, \gamma_{c}}\left(r_{s d}\right)+\frac{R_{c}}{2} \mathcal{P}_{O D, \gamma_{c}}\left(r_{s} d\right),
$$

where $R_{c}$ is the spectral efficiency in bits per second per hertz for cooperative transmission, and $\mathcal{P}_{O D, \gamma_{c}}\left(r_{s d}\right)$ denotes the outage probability for the direct link at rate $R_{c}$. In (44), note that one time slot is utilized if the direct link is not in outage, and two time slots are utilized if it is in outage. Note that the latter scenario is true even if the relay does not transmit because the time slot is wasted anyway. Averaging over the source-destination separation, the average rate is given by

$$
\bar{R}_{c}=\frac{R_{c}}{2}\left(1+\frac{2}{\alpha \rho^{2}}\left(\frac{K P}{\gamma_{c} N_{o}}\right)^{\frac{2}{\alpha}} \Gamma\left(\frac{2}{\alpha}, \frac{N_{o} \gamma_{c} \rho^{\alpha}}{K P}\right)\right) .
$$

We need to calculate the SNR threshold $\gamma_{c}$ corresponding to transmitting at rate $R_{c}$. The resulting SNR threshold $\gamma_{c}$ should generally be larger than $\gamma_{n c}$ required for noncooperative transmission. It is, in general, very difficult to find an explicit relation between the SNR threshold $\gamma_{c}$ and the transmission rate $R_{c}$, and thus, we render to a special case to capture the insights of this scenario. Let the outage be defined as the event that the mutual information $I$ between two terminals is less than some specific rate $R$ [20]. If the transmitted signals are Gaussian, then according to our channel model, the mutual information is given by $I=\log \left(1+\mathrm{SNR}_{s d}\right)$. The outage event for this case is defined as

$$
O_{I} \triangleq\left\{h_{s d}: I<R\right\}=\left\{h_{s d}: \mathrm{SNR}_{s d}<2^{R}-1\right\} .
$$

The above equation implies that if the outage is defined in terms of the mutual information and the transmitted signals are Gaussian, then the SNR threshold $\gamma_{c}$ and the spectral efficiency $R$ are related as $\gamma_{c}=2_{c}^{R}-1$, i.e., they exhibit an exponential relation. ${ }^{1}$ For the sake of comparison, $\bar{R}_{c}$ should be equal to $R$, the spectral efficiency of direct transmission. Thus, for a given $R$, one should solve for $R_{c}$. This can lead to many solutions for $R_{c}$, and we are going to choose the minimum $R_{c}$ [3]

In the following simulation comparisons, we study the outage probability performance when varying three basic quantities in our communication setup: the transmission rate, the transmit power, and the cell radius. In all the scenarios, we consider direct transmission, nearest neighbor, fixed relaying with six relays deployed in the network, and the Genie-aided lower bound. For all the cooperative transmission cases, both MRC and no-MRC are examined. In all the numerical results, the number of users is taken to be 100 , the propagation path loss is 3 , and the noise power density is $N_{o}=10^{-12}$. Simulation parameters are summarized in Table 1.

Fig. 5 depicts the outage performance versus the transmit power in decibel watt for a fixed cell radius of $1 \mathrm{~km}$. The spectral efficiency is taken to be $1 \mathrm{~b} / \mathrm{s} / \mathrm{Hz}$. . From the figure, we can see that cooperative diversity yields more steeper curves compared to direct transmission because of the spatial diversity gains. At an outage probability of 0.01 , there is more than $6 \mathrm{dbW}$ savings in the transmit power by using the nearest-neighbor protocol without MRC. With MRC, the gain increases to more than $7.5 \mathrm{dBW}$.

Fig. 6 depicts the results of the outage probability versus the cell radius when fixing the transmit power at $0.1 \mathrm{~W}$, and the spectral efficiency at $2 \mathrm{~b} / \mathrm{s} / \mathrm{Hz}$; see Table 1 for summary of simulation parameters. We can see that for outage probability less than 0.01 , we can have more than 200 percent increase in the cell size by using cooperative communications. These results reveal that cooperation is indeed a potential candidate for cell coverage increase

1. Intuitively, under a fixed modulation scheme and fixed average power constraint, one can think of the SNR threshold as being proportional to the minimum distance between the constellation points, which, in turn, depends on the number of constellation points for fixed average power, and the latter has an exponential relation to the number of bits per symbol that determines the spectral efficiency $R$. 
TABLE 1

SIMULATION PARAMETERS

\begin{tabular}{|c|c|}
\hline Parameter & Value \\
\hline Noise Power & $-90 \mathrm{dBm}$ \\
\hline Channel Fading & Zero-mean unit variance complex Gaussian \\
\hline Number of users dropped in the cell & 100 \\
\hline Pathloss exponent & 3 \\
\hline Cell Radius [Fig. 5] & $1 \mathrm{~km}$ \\
\hline Cell Radius [Fig. 7] & $2 \mathrm{~km}$ \\
\hline Modulation and Coding [Fig. 5] & QPSK rate 1/2 \\
\hline Modulation and Coding [Fig. 6] & QPSK rate 1 \\
\hline Transmit Power [Fig. 6, 7] & 20dBm (-10dBW) \\
\hline
\end{tabular}

without the need for extra base stations or extra transmit power. From the figure, we notice that the performance gap between direct transmission and cooperation diminishes with increasing the cell size. The reason for that is the increase in the probability of the outage event in the first transmission phase, which means a higher probability that the relay utilizes the second time slot for transmission, i.e., higher spectral efficiency loss.

Finally, Fig. 7 depicts the outage probability results versus the spectral efficiency in bits per second per hertz for a fixed cell size of $2 \mathrm{~km}$ and fixed transmit power of $0.1 \mathrm{~W}$, as indicated in Table 1 . It can be seen from the figure that through cooperation, the system can be operating at $2 \mathrm{~b} / \mathrm{s} / \mathrm{Hz}$ higher spectral efficiency than direct transmission at 1 percent outage probability. This translates to the capability of transmitting at higher date rates via cooperation without the need of increasing the transmit power, and still achieving the same quality of service as direct transmission.

In the numerical results, we can see that the infrastructure-based relay-assignment protocol has better performance than the nearest-neighbor protocol. The rationale is

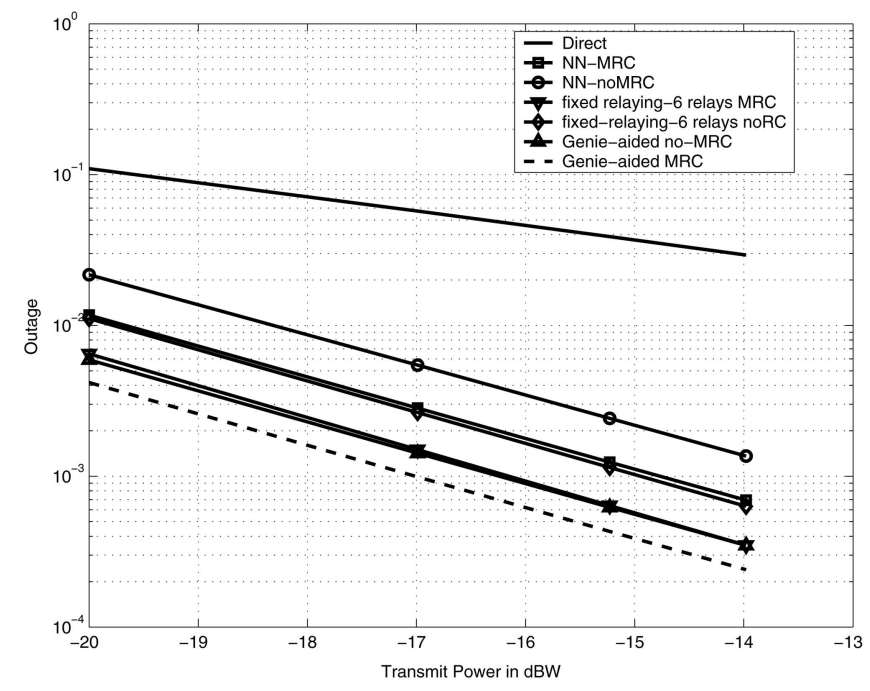

Fig. 5. Average outage probability versus the transmit power. that with the infrastructure-based protocol, there is higher probability that the fixed relay is located closer to the optimal point to the node than in the nearest-neighbor case where the relay is selected to be the closest node to the source. However, if users are willing to cooperate, for example, all nodes belong to the same authority or have common goal, then nearest neighbor is simpler to implement without the need of deploying relays in the network. Thus, there is a performance-complexity tradeoff.

\section{Conclusions}

In this paper, we addressed the relay-assignment problem for coverage extension in cooperative transmission over wireless networks based on the knowledge of the channel statistics governed by the users' spatial distribution. We proposed two distributed relay-assignment protocols. The Nearest neighbor is a simple algorithm in which the relay is selected to be the nearest neighbor to the user. We also considered the scenario where fixed relays are deployed in the network to help the existing users. Outage performance of the proposed protocols was analyzed. We further developed lower bounds on the performance of

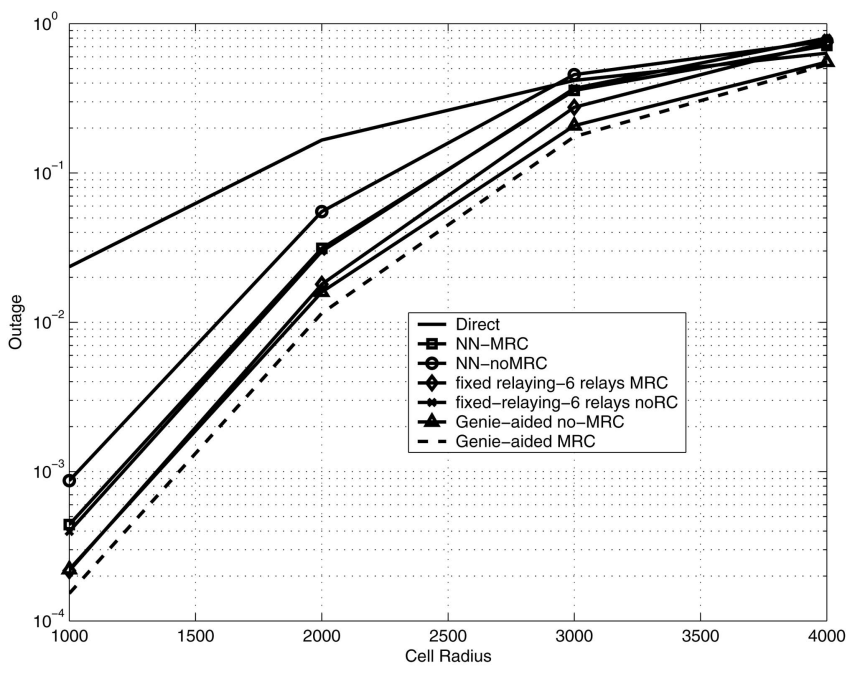

Fig. 6. Average outage probability versus the cell radius. 


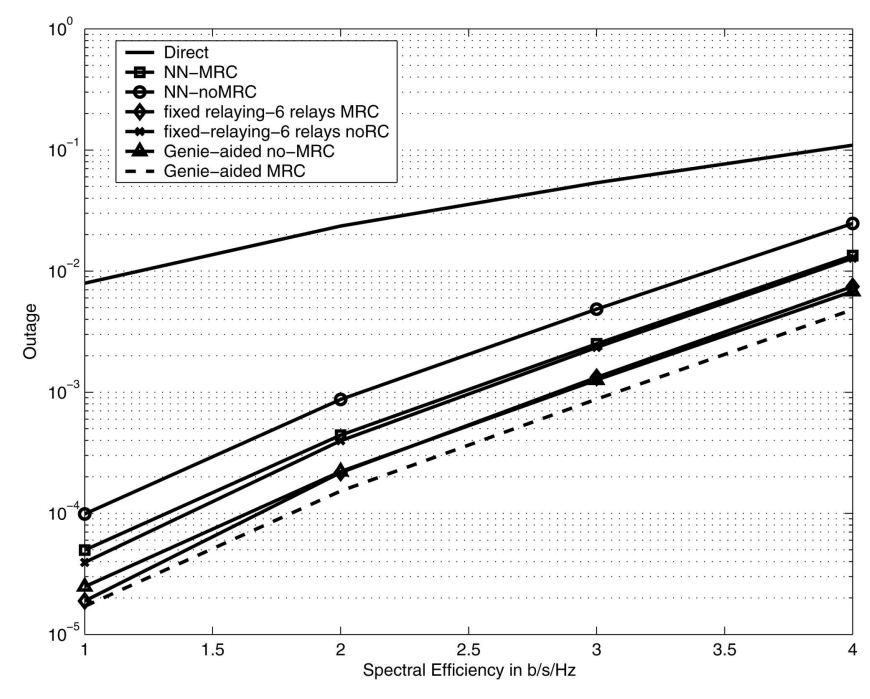

Fig. 7. Average outage probability versus the spectral efficiency.

any relay-assignment protocol via a Genie-aided method. Our numerical results indicate significant gains in the system performance. In particular, fixing the average transmit power, significant increase in the coverage area (more than 200 percent) of the network can be achieved by our simple distributed protocols. Similarly, for fixed cell radius, the average power required to achieve a certain outage probability is significantly reduced by more than $7 \mathrm{dbW}$ in our numerical examples. We have also shown that cooperation can allow, at the same quality of service, transmitting at higher rates compared to direct transmission; more than $2 \mathrm{~b} / \mathrm{s} / \mathrm{Hz}$ can be gained at the same transmit power. Our results also show that for larger cell sizes, the performance gap between direct and cooperative transmission diminishes.

\section{ACKNOWLEDGMENTS}

This work was supported in part by grants CTA-ARL DAAD 190120011, MURI AFOSR F496200210217, and US National Science Foundation CNS-0910461.

\section{REFERENCES}

[1] G.J. Foschini and M. Gans, "On the Limits of Wireless Communication in a Fading Environment when Using Multiple Antennas," Wireless Personal Comm., vol. 6, pp. 311-335, Mar. 1998.

[2] E. Telatar, "Capacity of Multi-Antenna Gaussian Channels," European Trans. Telecomm. vol. 10, pp. 585-595, Nov. 1999.

[3] J.N. Laneman, D.N.C. Tse, and G.W. Wornell, "Cooperative Diversity in Wireless Networks: Efficient Protocols and Outage Behavior," IEEE Trans. Information Theory, vol. 50, no. 12, pp. 30623080, Dec. 2004.

[4] J.N. Laneman and G.W. Wornell, “Distributed Space-Time Coded Protocols for Exploiting Cooperative Diversity in Wireless Networks," IEEE Trans. Information Theory, vol. 49, no. 10, pp. 24152525, Oct. 2003.

[5] A. Sendonaris, E. Erkip, and B. Aazhang, "User Cooperation Diversity-Part I: System Description," IEEE Trans. Comm., vol. 51, no. 11, pp. 1927-1938, Nov. 2003.

[6] G. Scutari and S. Barbarossa, "Distributed Space-Time Coding for Regenerative Relay Networks," IEEE Trans. Wireless Comm., vol. 4, no. 5, pp. 2387-2399, Sept. 2005.

[7] W. Su, A.K. Sadek, and K.J.R. Liu, "SER Performance Analysis and Optimum Power Allocation for Decode-and-Forward Cooperation Protocol in Wireless Networks," Proc. IEEE Wireless Comm. and Networking Conf. (WCNC), vol. 2, pp. 984-989, Mar. 2005.
[8] A.K. Sadek, W. Su, and K.J.R. Liu, "A Class of Cooperative Communication Protocols for Multi-Node Wireless Networks," Proc. IEEE Int'l Workshop Signal Processing Advances in Wireless Comm. (SPAWC), pp. 560-564, June 2005.

[9] A.K. Sadek, W. Su, and K.J.R. Liu, "Multinode Cooperative Communications in Wireless Networks," IEEE Trans. Signal Processing, vol. 55, no. 1, pp. 341-355, Jan. 2007.

[10] J. Luo, R.S. Blum, L.J. Greenstein, L.J. Cimini, and A.M. Haimovich, "New Approaches for Cooperative Use of Multiple Antennas in Ad-Hoc Wireless Networks," Proc. IEEE Vehicular Technology Conf. (VTC)-Fall, vol. 4, pp. 2769-2773, Sept. 2004.

[11] M. Dianati, X. Ling, S. Naik, and X. Shen, "A Node Cooperative ARQ Scheme for Wireless Ad Hoc Networks," IEEE Trans. Vehicular Technology, vol. 55, no. 3, pp. 1032-1044, May 2006.

[12] K.J.R. Liu, A.K. Sadek, W. Su, and A. Kwasinski, Cooperative Communications and Networking. Cambridge Univ. Press, 2008.

[13] Z. Han and K.J.R. Liu, Resource Allocation for Wireless Networks: Basics, Techniques, and Applications. Cambridge Univ. Press, 2008.

[14] A. Bletsas, A. Lippman, and D.P. Reed, "A Simple Distributed Method for Relay Selection in Cooperative Diversity Wireless Networks Based on Reciprocity and Channel Measurements," Proc. IEEE Vehicular Technology Conf. (VTC)-Spring, 2005.

[15] Y. Chen, G. Yu, P. Qiu, and Z. Zhang, "Power-Aware Cooperative Relay Selection Strategies in Wireless Ad Hoc Networks," Proc. IEEE Int'l Symp. Personal, Indoor and Mobile Radio Comm. (PIMRC), 2006.

[16] T.W. Ban, B.C. Jung, D.K. Sung, and W. Choi, "Performance Analysis of Two Relay Selection Schemes for Cooperative Diversity," Proc. IEEE Int'l Symp. Personal, Indoor and Mobile Radio Comm. (PIMRC), 2007.

[17] Y. Shi, S. Sharma, and Y. Thomas Hou, "Optimal Relay Assignment for Cooperative Communications," Proc. ACM MobiHoc, 2008.

[18] O. Dogan and S.G. Wilson, "Outage Probability and Assignment Protocols in Cooperative Diversity," Proc. IEEE Military Comm. Conf. (MILCOM), pp. 1-4, Oct. 29-31 2007.

[19] A.K. Sadek, Z. Han, and K.J.R. Liu, "A Distributed RelayAssignment Algorithm for Cooperative Communications in Wireless Networks," Proc. IEEE Int'l Conf. Comm. (ICC '06), vol. 4, pp. 1592-1597, June 2006.

[20] L.H. Ozaroq, S. Shamai, and A.D. Wyner, "Information Theoretic Considerations for Cellular Mobile Radio," IEEE Trans. Vehicular Technology, vol. 43, no. 5, pp. 359-378, May 1994.

[21] G. Sun, J. Chen, W. Guo, and K.J.R. Liu, "Signal Processing Techniques in Network-Aided Positioning: A Survey," IEEE Signal Processing Magazine, vol. 22, no. 4, pp. 12-23, July 2005.

[22] A.H. Sayed, A. Tarighat, and N. Khajehnouri, "Network-Based Wireless Location," IEEE Signal Processing Magazine, vol. 22, no. 4, pp. 24-40, July 2005.

[23] I.S. Gradshteyn and I.M. Ryzhik, Table of Integrals, Series, and Products, fourth ed. Academic Press, 1980.

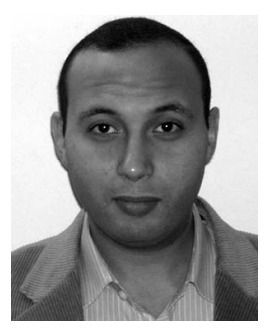

Ahmed K. Sadek received the BSc degree (with highest Honors) and the MSc degree in electrical engineering from Alexandria University, Egypt, in 2000 and 2003, respectively, and the PhD degree in electrical engineering from the University of Maryland, College Park, in 2007. He is currently a senior engineer with Corporate Research and Development, Qualcomm Incorporated. His research interests include information theory, communication theory, and signal processing with current focus on cognitive radios, spectrum sharing, interference management, and cooperative communications. In 2000, he received the first prize in the IEEE Egypt Section undergraduate student contest for the BSc graduation project. He received the Graduate School Fellowship from the University of Maryland in 2003 and 2004, and the Distinguished Dissertation Fellowship Award from the Department of Electrical Engineering, University of Maryland in 2007. $\mathrm{He}$ is a coauthor of Cooperative Communications and Networking, Cambridge University Press, 2008. He is a member of the IEEE. 


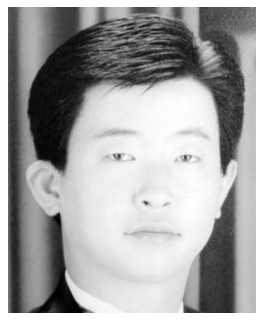

Zhu Han received the BS degree in electronic engineering from Tsinghua University, and the MS and PhD degrees in electrical engineering from the University of Maryland, College Park, in 1997, 1999, and 2003, respectively. From 2000 to 2002, he had been an R\&D engineer of JDSU, Germantown, Maryland. From 2002 to 2003, he was a graduate research assistant at the University of Maryland. From 2003 to 2006, he was a research associate at the University of Maryland. From 2006 to 2008, he was an assistant professor at Boise State University, Idaho. Currently, he is an assistant professor in the Electrical and Computer Engineering Department at the University of Houston, Texas. During June-August 2006, he was a visiting scholar at Princeton University. During May-August 2007, he was a visiting professor at Stanford University. During May-August 2008, he was a visiting professor at the University of Oslo, Norway, and Supelec, Paris, France. In July 2009, he was a visiting professor at the University of Illinois at Urbana-Champagne. His research interests include wireless resource allocation and management, wireless communications and networking, game theory, wireless multimedia, and security. He is the MAC Symposium vice chair of the IEEE Wireless Communications and Networking Conference, 2008. He is the guest editor for the Special Issue on Fairness of Radio Resource Management Techniques in Wireless Networks, EURASIP Journal on Wireless Communications and Networking, and Special Issue on Game Theory, EURASIP Journal on Advances in Signal Processing. He is the coauthor for the papers that won the Best Paper Awards at the IEEE International Conference on Communications 2009 and the Seventh International Symposium on Modeling and Optimization in Mobile, Ad Hoc, and Wireless Networks (WiOpt '09). He is a senior member of the IEEE.

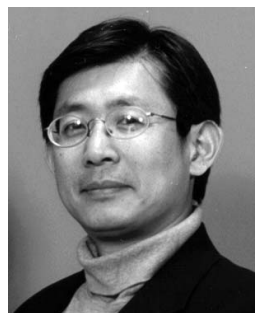

K.J. Ray Liu is a distinguished scholar-teacher of the University of Maryland, College Park. He is an associate chair of graduate studies and research of the Electrical and Computer Engineering Department and leads the Maryland Signals and Information Group conducting research encompassing broad aspects of information technology including communications and networking, information forensics and security, multimedia signal processing, and biomedical technology. He is the recipient of numerous honors and awards including Best Paper Awards from the IEEE Signal Processing Society, the IEEE Vehicular Technology Society, and the EURASIP. He is also an IEEE Signal Processing Society distinguished lecturer and received the EURASIP Meritorious Service Award and the US National Science Foundation Young Investigator Award. He also received various teaching and research recognitions from the University of Maryland, including the university-level Invention of the Year Award, and the Poole and Kent Senior Faculty Teaching Award and Outstanding Faculty Research Award, both from A. James Clark School of Engineering. He is a fellow of the IEEE and the AAAS. He was vice president-publications and will serve as president-elect from 2010. He was the editor-in-chief of the IEEE Signal Processing Magazine and the founding editor-in-chief of the EURASIP Journal on Applied Signal Processing. His recent books include Cooperative Communications and Networking, Cambridge University Press, 2008; Resource Allocation for Wireless Networks: Basics, Techniques, and Applications, Cambridge University Press, 2008; Ultra-Wideband Communication Systems: The Multiband OFDM Approach, IEEE-Wiley, 2007; Network-Aware Security for Group Communications, Springer, 2007; Multimedia Fingerprinting Forensics for Traitor Tracing, Hindawi, 2005; Handbook on Array Processing and Sensor Networks, IEEE-Wiley, 2009.

$\triangleright$ For more information on this or any other computing topic, please visit our Digital Library at www.computer.org/publications/dlib. 